\title{
Long-term effects of a randomised trial of a 6-year lifestyle intervention in impaired glucose tolerance on diabetes-related microvascular complications: the China Da Qing Diabetes Prevention Outcome Study
}

\author{
Q. Gong • E. W. Gregg • J. Wang • Y. An • P. Zhang $\cdot$ W. Yang $\cdot$ H. Li $\cdot$ H. Li $\cdot$ \\ Y. Jiang $\cdot$ Y. Shuai $\cdot$ B. Zhang $\cdot$ J. Zhang $\cdot$ R. B. Gerzoff • G. Roglic $\cdot$ Y. Hu $\cdot$ G. Li $\cdot$ \\ P. H. Bennett
}

Received: 24 May 2010 /Accepted: 20 September 2010 /Published online: 3 November 2010

(C) Springer-Verlag 2010

\begin{abstract}
Aims/hypothesis We determined the effects of 6 years of lifestyle intervention in persons with impaired glucose tolerance (IGT) on the development of retinopathy, nephropathy and neuropathy over a 20 year period.

Methods In 1986, 577 adults with IGT from 33 clinics in Da Qing, China were randomly assigned by clinic to a control group or one of three lifestyle intervention groups (diet, exercise, and diet plus exercise). Active intervention was carried out from 1986 to 1992 . In 2006 we conducted a 20 year follow-up study of the original participants to
\end{abstract}

Q. Gong $\cdot$ Y. An $\cdot$ W. Yang $\cdot$ H. Li $\cdot$ Y. Shuai $\cdot$ B. Zhang $\cdot$

G. Li $(\triangle)$

Department of Endocrinology, China-Japan Friendship Hospital,

Yinghua Dong Lu, Chaoyang District,

Beijing 100029, China

e-mail: guangwei_li@medmail.com.cn

E. W. Gregg • P. Zhang $\cdot$ R. B. Gerzoff

Division of Diabetes Translation,

Centers for Disease Control and Prevention,

Atlanta, GA, USA

J. Wang $\cdot$ H. Li $\cdot$ Y. Jiang $\cdot$ J. Zhang $\cdot$ Y. Hu

Department of Cardiology, Da Qing First Hospital,

Da Qing, China

G. Roglic

Department of Chronic Diseases and Health Promotion,

World Health Organization,

Geneva, Switzerland

Q. Gong $\cdot$ P. H. Bennett

Diabetes Epidemiology and Clinical Research Section,

National Institute of Diabetes and Digestive and Kidney Diseases,

National Institutes of Health,

Phoenix, AZ, USA compare the incidence of microvascular complications in the combined intervention group vs the control group.

Results Follow-up information was obtained on 542 (94\%) of the 577 original participants. The cumulative incidence of severe retinopathy was $9.2 \%$ in the combined intervention group and $16.2 \%$ in the control group $(p=0.03$, logrank test). After adjusting for clinic and age, the incidence of severe retinopathy was $47 \%$ lower in the intervention group than the control group (hazard rate ratio $0.53,95 \%$ CI $0.29-0.99, p=0.048)$. No significant differences were found in the incidence of severe nephropathy (hazard rate ratio $1.05,95 \%$ CI $0.16-7.05$, intervention vs control, $p=$ $0.96)$ or in the prevalence of neuropathy $(8.6 \%$ vs $9.1 \%, p=$ 0.89 ) among the 20 year survivors.

Conclusions/interpretation Lifestyle intervention for 6 years in IGT was associated with a $47 \%$ reduction in the incidence of severe, vision-threatening retinopathy over a 20 year interval, primarily due to the reduced incidence of diabetes in the intervention group. However, similar benefits were not seen for nephropathy or neuropathy.

Keywords Diabetic retinopathy Impaired glucose tolerance $\cdot$ Lifestyle intervention $\cdot$ Nephropathy $\cdot$ Neuropathy

$\begin{array}{ll}\text { Abbreviations } \\ \text { ACR } & \text { Albumin to creatinine ratio } \\ \text { CDQDPOS } & \text { China Da Qing Diabetes Prevention } \\ & \text { Outcome Study } \\ \text { CDQDPS } & \text { China Da Qing Diabetes Prevention Study } \\ \text { ESRD } & \text { End-stage renal disease } \\ \text { IGT } & \text { Impaired glucose tolerance } \\ \text { NPR } & \text { Non-proliferative retinopathy } \\ \text { PR } & \text { Proliferative retinopathy }\end{array}$




\section{Introduction}

Several studies have shown that lifestyle or pharmacological interventions can reduce diabetes incidence in people with impaired glucose tolerance (IGT) [1-7]. In the China Da Qing Diabetes Prevention Study (CDQDPS), lifestyle intervention among people with IGT over a 6 year period reduced the incidence of diabetes by $33 \%$ in the diet only group, $47 \%$ in the exercise group and $38 \%$ in the diet plus exercise group compared with the control group, but there was no significant difference among the three intervention groups [1]. Follow-up over a 20 year period showed an overall $43 \%$ reduction in diabetes incidence in the combined intervention group compared with the control group [2].

Diabetes mellitus leads to both macro- and microvascular complications that are responsible for most of the associated excess morbidity and mortality. While macrovascular complications such as cardiovascular disease are the most frequent cause of excess mortality, microvascular complications such as retinopathy and nephropathy are responsible for much of the excess morbidity [8-15]. Despite the perceived value of lifestyle interventions, whether the reduced incidence of diabetes also translates to a reduced incidence of microvascular complications is unclear. We have previously reported that the lifestyle intervention in this study resulted in a non-significant reduction in first cardiovascular disease (CVD) events (hazard rate ratio $0.98,95 \%$ CI $0.71-1.37$ ), CVD mortality (hazard rate ratio $0.83,95 \%$ CI $0.48-1.40$ ) and all-cause mortality (hazard rate ratio $0.96,95 \%$ CI $0.65-1.41$ ) [2]. Whether or not lifestyle interventions influence these events remains uncertain because of the limited power of the present study to detect such changes.

Here we report the results of a follow-up study of CDQDPS participants on the effects of lifestyle intervention on the development of retinopathy, nephropathy and neuropathy over a 20 year period.

\section{Methods}

Design overview The design and conduct of the CDQDPS and the outcome study (CDQDPOS) have been described previously $[1,2,16]$. In brief, the CDQDPS was conducted between 1986 and 1992 to test whether lifestyle intervention can reduce diabetes incidence in Chinese people with IGT. In 1986, 577 people with IGT from 33 outpatient clinics in Da Qing, China were randomised by clinic to either a control group or one of three lifestyle intervention groups (diet, exercise, and diet plus exercise). The goal of the diet intervention was to increase participants' vegetable intake and lower their alcohol and sugar intake and, in those who were overweight or obese, to lose weight by reducing total calorie intake. The goal of the exercise intervention was to increase leisure-time physical activity. The intervention took place over 6 years after which the participants were informed of their individual test results and told about the reduced incidence of diabetes among those who received lifestyle interventions. All participants were then asked to continue with usual medical care.

In 2006, a follow-up study (CDQDPOS) of the original participants 20 years after randomisation was conducted to determine the effect of the 6 year intervention on diabetes incidence and on prespecified diabetes-related complications, including microvascular disease. As diabetes incidence did not differ significantly among the three intervention groups during the active intervention period and because of limited power to detect differences, the intervention groups were combined and comparisons were made between the combined intervention group and the control group. The characteristics of study participants at baseline, the end of the 6 year active intervention and the end of the 20 year follow-up have been reported elsewhere [1, 2]. The institutional review boards of WHO and the China-Japan Friendship Hospital approved the protocol. All study participants or their proxies who provided information about deceased participants gave written informed consent.

Data collection In 1986, 110,660 residents aged $\geq 25$ years were screened in Da Qing, China, by measurement of plasma glucose concentration after a standard breakfast (a steamed bun containing $\geq 80 \mathrm{~g}$ carbohydrate), and 3,956 participants with $2 \mathrm{~h}$ plasma glucose $\geq 6.67 \mathrm{mmol} / 1$ after breakfast were given a 75 g OGTT test; 577 of the tested participants had IGT. Baseline examinations, questionnaires, demographic information were collected, and retinal examinations were performed by two ophthalmologists using direct ophthalmoscopy. Pupils were dilated and fundus photographs were taken of each eye. Of the participants with IGT, $14.7 \%$ had non-proliferative retinopathy but none had proliferative retinopathy [16].

In 2006, data on the living participants were collected by personal interview, medical chart review and clinical examination. For deceased participants, data were obtained by proxy interview and medical chart review. Dates of retinal photocoagulation, onset of blindness, renal replacement treatment, amputation and death were determined by interviews and validated by medical chart review. Trained abstracters, masked to the participants' intervention status, reviewed the medical records.

Clinical examinations of living participants were performed by examiners who were also not aware of the participants' intervention status. These examinations included measurement of height, weight and inspection of lower extremities for ulcers and amputations. Sitting brachial BP 
was measured twice using a standard mercury sphygmomanometer and the average of the readings was recorded. Brachial and posterior tibial and/or dorsalis pedis BP was measured using Doppler ultrasound. The ankle-brachial index was calculated and the lower value from either side was used. A $45^{\circ}$ single-field colour fundus photograph of each eye was taken with a Canon non-mydriatic retinal camera using a standardised photography protocol, and slit-lamp biomicroscopy was used in those whose retina could not be visualised or who had unsatisfactory photographs. The retinal photographs were graded independently for the presence and severity of diabetic retinopathy by two ophthalmologists (L. Zhang and H. Zhang) who were masked to the participants' intervention status. Disagreements between the readers were referred to an independent reader for arbitration. The graders used a modification of the Early Treatment Diabetic Retinopathy Study grading system [17]. A test of sensory neuropathy was performed in which a $10 \mathrm{~g}$ monofilament was placed perpendicular to five designated sites in each foot (1st toe, 4th toe and over 1st, 3rd and 5th metatarsal joints on the ventral surface), avoiding callosities, and pressed until it bent, and the participants were asked if they could feel the touch [18]. After an overnight fast (8$12 \mathrm{~h}$ ), blood and urine specimens were obtained and a $75 \mathrm{~g}$ load OGTT was administered. Blood specimens were centrifuged immediately after collection, plasma glucose was determined by a glucose oxidase method, $\mathrm{HbA}_{1 \mathrm{c}}$ by latex immunoagglutination, serum and urine creatinine by enzyme method and urinary albumin by immunonephelometry. The ratio of urinary albumin to creatinine (ACR) was calculated.

Outcome classification and assessment Retinopathy status was determined from a history of retinal photocoagulation or blindness, and retinal photographs or slit-lamp examination. Abnormalities on the retinal photographs or from the slit-lamp examination were classified according to changes in the worse affected eye. Non-proliferative retinopathy (NPR) was defined as microaneurysm(s) and/or intraretinal haemorrhage(s) and/or hard exudates and/or cotton wool spots and/or venous beading. Proliferative retinopathy (PR) was defined as new vessels and/or fibrous proliferation and/ or vitreous haemorrhage and/or retinal detachment and/or scars of photocoagulation in either eye.

Severe retinopathy was defined as a history of photocoagulation, blindness or PR. The date of onset of severe retinopathy was taken as the date of initiation of retinal photocoagulation or occurrence of blindness according to the chart review or interview, or as 2006 if it was defined only by photographic or slit-lamp examination. One participant who was blind at the time of randomisation and one participant whose photocoagulation date was unknown were excluded.

Nephropathy was defined as a history of renal dialysis or transplantation, death from nephropathy or end-stage renal disease (ESRD), or among living participants as ACR $\geq 300 \mathrm{mg} / \mathrm{g}$ (to convert values to $\mathrm{mg} / \mathrm{mmol}$ multiply by $0.113)$ or serum creatinine $\geq 177 \mu \mathrm{mol} / \mathrm{l}(2 \mathrm{mg} / \mathrm{dl})$. Severe nephropathy was defined as that which led to renal replacement therapy or death from nephropathy or ESRD.

Overt neuropathy was defined as a history of lower extremity amputation, gangrene or ulceration. In living participants, abnormal touch sensation was defined as loss of sensation at one or more sites in the monofilament test.

Diabetes status was defined using the 1985 WHO criteria [19] from results of the OGTT done every 2 years during the active intervention period (1986-1992) or at the end of the study (2006), or by self-reported physiciandiagnosed diabetes with evidence of elevated glucose levels in the medical record, or as taking hypoglycaemic medications, as described previously $[1,2]$. The date of diagnosis of diabetes was taken as its date of first recognition.

Statistical analysis To determine the incidence of outcome events, data were censored at the date of first report of the specific event, death, loss to follow-up or 31 December 2006, whichever came first. The incidence of events was computed as the number of cases per 1,000 person-years at risk. Confidence intervals for incidence density were calculated using Poisson distribution estimates [20].

Using intention-to-treat analysis, the development of severe retinopathy and nephropathy in the intervention and control group was compared by the Kaplan-Meier method; $p$ values were computed by log-rank tests and hazard rate ratios comparing the rates in the intervention group with those in the control group adjusted for age and clinicclustering were estimated by Cox proportional hazards analysis (NLMIXED), which fits non-linear mixed models by maximising an approximation to the likelihood integrated over the random effects. The number needed to treat was calculated as described by Altman and Andersen [21]. The prevalences of retinopathy, macro- or microalbuminuria and neuropathy were calculated for those alive in 2006. The statistical significance of differences between groups was assessed using the $\chi^{2}$ test for categorical and the $t$-test for continuous variables. The Mantel-Haenszel $\chi^{2}$ test for trend was used to evaluate the effects of follow-up and diabetes duration. Results were considered significant if $p<$ 0.05. Statistical analyses were performed using SAS System for Windows, version 9.1 (SAS Institute Inc, Cary NC, USA).

\section{Results}

The flow chart of the follow-up study is shown in Fig. 1. In 2006, of 577 original participants, information on one or more of the complications was obtained in 542 (94\%). The 


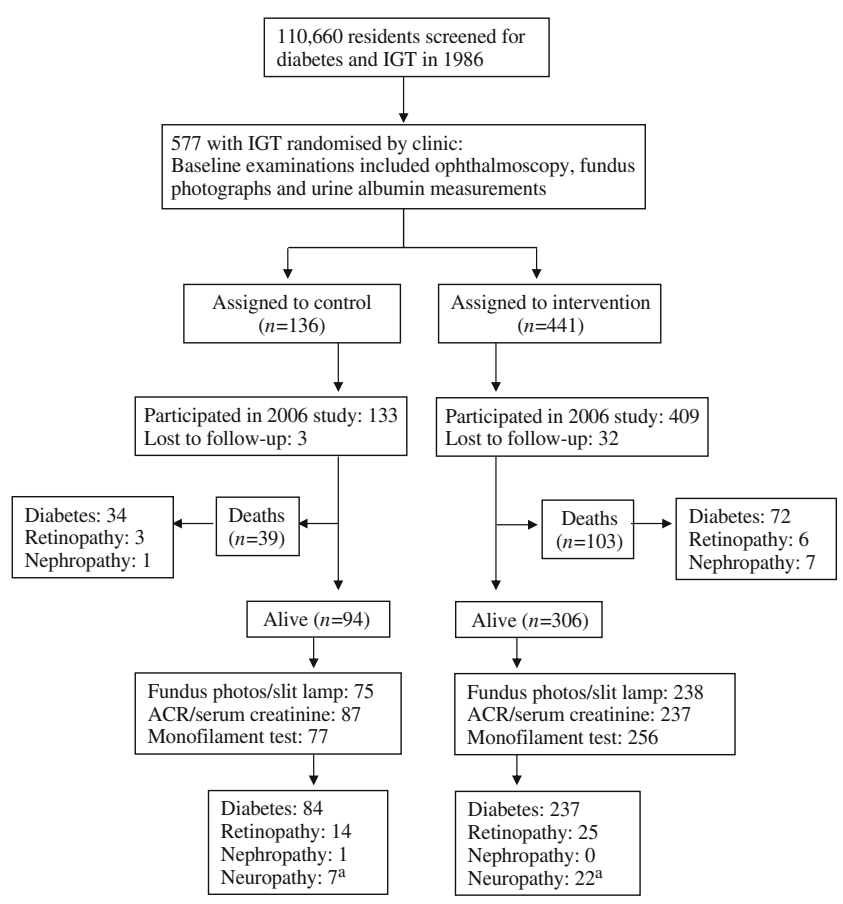

Fig. 1 Flow diagram of the China Da Qing Diabetes Prevention Outcome Study showing assignments to control and intervention groups, losses to follow-up and the main sources of information on retinopathy, nephropathy and neuropathy among participants. The numbers of participants who developed diabetes and each of the

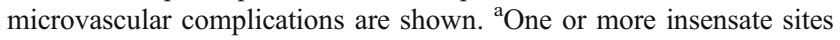
by the monofilament test

characteristics of the participants at baseline and among living participants at the 20 year follow-up in 2006 are shown in Table 1. Comparisons of the baseline characteristics of the living participants examined in 2006 and those unavailable for examination showed no significant difference between the groups.

Retinopathy Information about eye disease was obtained on $540(94 \%)$ of the original participants, of whom 48 had severe retinopathy. Severe retinopathy occurred in 31 participants in the intervention group and 17 in the control group, leading to a cumulative incidence of 9.2 and $16.2 \%$, respectively ( $p=0.03$, log-rank test; Fig. 2 ). In proportional hazards analysis with adjustment for age and clinicclustering, the intervention group had a $47 \%$ lower incidence of severe retinopathy than the control group (hazard rate ratio $0.53,95 \%$ CI $0.29-0.99, p=0.048$ ). There were no significant differences in the incidence of severe retinopathy among participants in the originally assigned intervention groups (diet only, 4.5/1,000 person-years, 95\% CI 1.8-7.1; exercise only $5.3 / 1,000$ person-years, $95 \%$ CI $2.5-8.0$; diet plus exercise $2.6 / 1,000$ person-years, $95 \%$ CI $0.5-4.6$ ).

The sources of information on severe retinopathy were as follows. Nine cases were reported among the 142 participants who had died, of whom four had a history of photocoagulation and five were blind. Among 400 survivors, 39 had severe retinopathy. Three hundred and one had gradable retinal photographs and in 12 objective information was obtained only from slit-lamp examination. These retinal examinations showed photocoagulation scars $(n=20)$

Table 1 Baseline and follow-up characteristics of study participants

\begin{tabular}{|c|c|c|}
\hline Variable & Intervention group & Control group \\
\hline Total number of participants & 441 & 136 \\
\hline \multicolumn{3}{|l|}{1986} \\
\hline Sex (men/women) & $235 / 206$ & $77 / 59$ \\
\hline Age (years) & $44.7 \pm 9.3$ & $46.7 \pm 9.2^{\mathrm{a}}$ \\
\hline BMI $\left(\mathrm{kg} / \mathrm{m}^{2}\right)$ & $25.6 \pm 4.0$ & $26.2 \pm 3.8$ \\
\hline \multicolumn{3}{|l|}{$\mathrm{BP}(\mathrm{mmHg})$} \\
\hline Systolic & $132.2 \pm 23.5$ & $134.4 \pm 23.4$ \\
\hline Diastolic & $87.2 \pm 13.5$ & $88.5 \pm 13.5$ \\
\hline $\begin{array}{l}\text { Fasting plasma glucose } \\
(\mathrm{mmol} / \mathrm{l})\end{array}$ & $5.6 \pm 0.8$ & $5.5 \pm 0.8$ \\
\hline $2 \mathrm{~h}$ Plasma glucose $(\mathrm{mmol} / \mathrm{l})$ & $9.0 \pm 0.9$ & $9.0 \pm 0.9$ \\
\hline \multicolumn{3}{|l|}{2006} \\
\hline Total number of participants & 306 & 94 \\
\hline BMI $\left(\mathrm{kg} / \mathrm{m}^{2}\right)$ & $24.5 \pm 3.1(n=266)$ & $24.4 \pm 3.5(n=82)$ \\
\hline $\mathrm{BP}(\mathrm{mmHg})$ & $(n=285)$ & $(n=87)$ \\
\hline Systolic & $144.6 \pm 23.6$ & $145.1 \pm 19.1$ \\
\hline Diastolic & $82.4 \pm 12.3$ & $82.8 \pm 11.5$ \\
\hline $\begin{array}{l}\text { Fasting plasma glucose } \\
(\mathrm{mmol} / \mathrm{l})\end{array}$ & $7.9 \pm 3.2(n=260)$ & $8.7 \pm 3.1(n=80)^{*}$ \\
\hline $\begin{array}{l}2 \text { h Plasma glucose } \\
(\mathrm{mmol} / \mathrm{l})\end{array}$ & $11.5 \pm 4.6(n=100)$ & $13.8 \pm 6.0(n=28)^{*}$ \\
\hline $\mathrm{HbA}_{1 \mathrm{c}}(\%)$ & $7.34 \pm 1.7(n=271)$ & $7.83 \pm 2.0(n=81)^{*}$ \\
\hline Diabetes status & $(n=306)$ & $(n=93)$ \\
\hline Normal glucose tolerance & $40(13.1)$ & $3(3.2)^{*}$ \\
\hline IGT & $29(9.5)$ & $6(6.5)^{*}$ \\
\hline Diabetes & $237(77.4)$ & $84(90.3)^{*}$ \\
\hline Retinopathy & $(n=238)$ & $(n=75)$ \\
\hline No retinopathy & $135(56.7)$ & $38(50.7)$ \\
\hline Non-proliferative retinopathy & $86(36.1)$ & $29(38.7)$ \\
\hline Proliferative retinopathy & $17(7.2)$ & $8(10.6)$ \\
\hline $\operatorname{ACR}(\mathrm{mg} / \mathrm{g})^{\mathrm{a}}$ & $(n=254)$ & $(n=79)$ \\
\hline$<30$ & $151(59.5)$ & $44(55.7)$ \\
\hline 30-299 & $93(36.6)$ & $32(40.5)$ \\
\hline$\geq 300$ & $10(3.9)$ & $3(3.8)$ \\
\hline \multicolumn{3}{|l|}{ Neuropathy $(n=333)$} \\
\hline$\geq 1$ Insensate site & $22(8.6)$ & $7(9.1)$ \\
\hline$\geq 3$ Insensate sites & $11(4.3)$ & $5(6.5)$ \\
\hline Ankle-brachial index & $(n=262)$ & $(n=79)$ \\
\hline$<0.9$ & $22(8.4)$ & $10(12.7)$ \\
\hline $0.9-1.5$ & $240(91.6)$ & $69(87.3)$ \\
\hline
\end{tabular}

Data are mean \pm SD or $n(\%)$

${ }^{\mathrm{a}}$ To convert values to $\mathrm{mg} / \mathrm{mmol}$ multiply by 0.113 $* p<0.05$ 


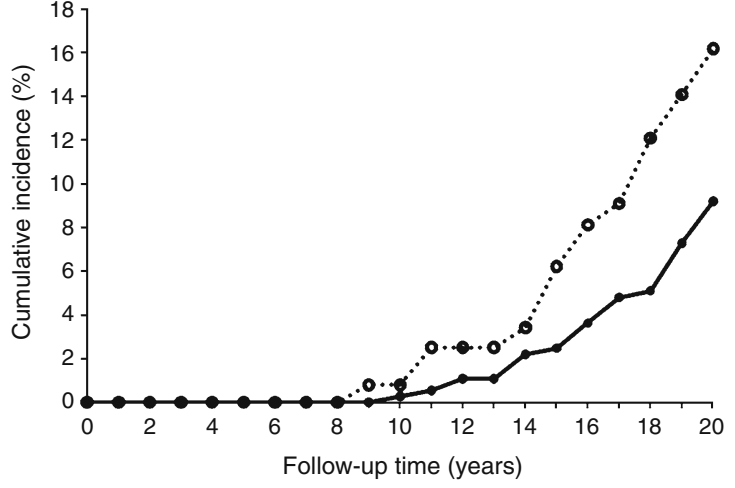

Number at risk:

$\begin{array}{llllllllllll}\text { Control } & 133 & 132 & 131 & 128 & 128 & 121 & 113 & 107 & 98 & 91 & 82\end{array}$

$\begin{array}{llllllllllll}\text { Intervention } & 407 & 407 & 402 & 393 & 387 & 376 & 364 & 355 & 339 & 316 & 290\end{array}$

Fig. 2 Cumulative incidence of severe retinopathy during the 20 year follow-up of the China Da Qing Diabetes Prevention Outcome Study in the control group (dotted line, white circles) and intervention group (solid line, black circles). The number at risk represents the number of participants who contributed to the cumulative incidence of severe retinopathy at each of the follow-up years in the control and intervention groups. The hazard rate ratio between the intervention and control group was 0.53 (95\% CI $0.29-0.99)$

or signs of proliferative retinopathy $(n=5)$. The medical chart reviews provided evidence of photocoagulation in the two others. Five had only a positive history (two blindness, three photocoagulation). Among the other 87 surviving participants without retinal examination, seven had severe retinopathy (one blindness, six photocoagulation), of whom one had a positive chart review and six were reported by history only. Among the 313 participants who had gradable fundus photographs or slit-lamp examination, the history of severe retinopathy obtained from the interview agreed with the objective assessment in $93.6 \%(\kappa=0.76)$.

The incidence of severe retinopathy increased as followup time increased, with 36 of the 48 events occurring during the last 5 years of follow-up ( $p_{\text {trend }}<0.0001$; Table 2 ). All those with severe retinopathy had developed diabetes by the time their retinopathy was recognised and the incidence rose rapidly as the duration of diabetes increased $\left(p_{\text {trend }}<0.0001\right.$; Table 3$)$. When adjusted for differences in the duration of diabetes between the intervention and control groups, the incidence in the two groups was similar (incidence rate ratio $0.85,95 \%$ CI $0.47-1.54$, intervention vs control, $p=0.59$ ), indicating that the difference in incidence of severe retinopathy in the intervention and control groups was largely explained by the lower incidence of diabetes and the mean delay of 3.6 years in the onset of diabetes in the intervention group [2].

Based on information from the retinal photographs or slit-lamp examinations, NPR was present in $36.1 \%$ in the intervention group and $38.7 \%$ in the control group ( $p=0.51$, Table 1). NPR was seen in $41.6 \%$ of those with diabetes and $14.3 \%$ of those without diabetes.

Nephropathy Information on nephropathy was obtained in $542(94 \%)$ of the original participants. Severe nephropathy requiring renal replacement therapy or leading to death occurred in nine participants (seven in the intervention group and two in the control group), all of whom had diabetes when they developed renal disease. Two had received renal dialysis (one in the intervention group, one in the control group) and one in the intervention group had a renal transplant. Six (five in the intervention group and one in the control group) died from diabetic nephropathy or ESRD. The cumulative incidence of severe nephropathy was $2.1 \%$ in the intervention group and $2.0 \%$ in the control group ( $p=0.95$, log-rank test), and in proportional hazards analysis the hazard rate ratio was 1.05 (intervention vs control, 95\% CI 0.16-7.05, $p=0.96$ ). Among living participants in $2006, \mathrm{ACR} \geq 300 \mathrm{mg} / \mathrm{g}$ or an elevated serum creatinine level was present only among those who had developed diabetes and was present in $4.0 \%$ in the intervention group and $4.6 \%$ in the control group $(p=0.82)$. Of the 15 with $\mathrm{ACR} \geq 300 \mathrm{mg} / \mathrm{g}$ or elevated creatinine,

Table 2 Incidence of severe retinopathy by follow-up time from randomisation

\begin{tabular}{|c|c|c|c|c|c|c|c|c|c|}
\hline \multirow{2}{*}{$\begin{array}{l}\text { Follow-up } \\
\text { time (years) }\end{array}$} & \multicolumn{4}{|c|}{ Intervention group } & \multicolumn{4}{|c|}{ Control group } & \multirow{2}{*}{$\begin{array}{l}\text { Hazard rate ratio } \\
(95 \% \mathrm{CI})\end{array}$} \\
\hline & $\begin{array}{l}\text { No. of } \\
\text { cases }\end{array}$ & $\begin{array}{l}\text { No. of } \\
\text { participants }\end{array}$ & $\begin{array}{l}\text { Person- } \\
\text { years }\end{array}$ & $\begin{array}{l}\text { Incidence } \\
\text { (/1,000 person- } \\
\text { years })\end{array}$ & $\begin{array}{l}\text { No. of } \\
\text { cases }\end{array}$ & $\begin{array}{l}\text { No. of } \\
\text { participants }\end{array}$ & $\begin{array}{l}\text { Person- } \\
\text { years }\end{array}$ & $\begin{array}{l}\text { Incidence } \\
\text { (/1,000 person- } \\
\text { years })\end{array}$ & \\
\hline $0-9.9$ & 0 & 439 & 4,067 & - & 1 & 136 & 1,306 & 0.77 & - \\
\hline $10-14.9$ & 8 & 378 & 1,796 & 4.45 & 3 & 121 & 549 & 5.46 & $0.82(0.22-3.10)$ \\
\hline $15-20$ & 23 & 348 & 1,579 & 14.6 & 13 & 104 & 454 & 28.6 & $0.51(0.26-1.01)$ \\
\hline Total & 31 & 439 & 7,442 & 4.2 & 17 & 136 & 2,301 & 7.4 & $0.53(0.29-0.99)^{\mathrm{a}, \mathrm{b}}$ \\
\hline
\end{tabular}

$p_{\text {trend }}<0.0001$ for follow-up time

${ }^{a}$ Adjusted for age and clinic-clustering

${ }^{\mathrm{b}}$ Intervention vs control group 
Table 3 Incidence of severe retinopathy by diabetes duration

\begin{tabular}{|c|c|c|c|c|c|c|}
\hline \multirow[b]{2}{*}{$\begin{array}{l}\text { Time since recognition } \\
\text { of diabetes (years) }\end{array}$} & \multicolumn{3}{|c|}{ Intervention group } & \multicolumn{3}{|c|}{ Control group } \\
\hline & $\begin{array}{l}\text { No. of } \\
\text { cases }\end{array}$ & Person-years & $\begin{array}{l}\text { Incidence } / 1,000 \\
\text { person-years }(95 \% \mathrm{CI})\end{array}$ & $\begin{array}{l}\text { No. of } \\
\text { cases }\end{array}$ & Person-years & $\begin{array}{l}\text { Incidence } 1,000 \\
\text { person-years }(95 \% \mathrm{CI})\end{array}$ \\
\hline $0-4.9$ & 3 & 1,332 & $2.3(0.5-6.6)$ & 1 & 503 & $2.0(0.05-11.1)$ \\
\hline $5-9.9$ & 10 & 1,031 & $9.7(4.7-17.8)$ & 4 & 410 & $9.8(2.7-25.0)$ \\
\hline $10-14.9$ & 7 & 665 & $10.5(4.2-21.7)$ & 8 & 303 & $26.4(11.4-52.0)$ \\
\hline $15-20$ & 11 & 127 & $86.6(43.2-155.0)$ & 4 & 79 & $50.6(13.8-129.6)$ \\
\hline
\end{tabular}

Duration-standardised incidence rate ratio (intervention vs control): 0.85 ( $95 \%$ CI $0.47-1.54, p=0.59$ )

eight had severe retinopathy. ACR in the microalbuminuric range $(30-299 \mathrm{mg} / \mathrm{g})$ was frequent $(36.6 \%$ in the intervention group and $40.5 \%$ in the control group, $p=0.82$; Table 1 ).

Neuropathy Overt neuropathy was uncommon. Amputations or gangrene were reported in four of the 542 participants. Foot ulcers were seen in four of 333 living participants with foot examinations, one of whom had amputation. All with overt neuropathy had already developed diabetes. Abnormal touch sensation with one or more insensate sites occurred in $29(8.7 \%)$, with similar prevalences in the intervention and control groups ( $8.6 \%$ and $9.1 \%$, respectively, $p=0.89$; Table 1 ).

\section{Discussion}

This study demonstrated that a 6-year lifestyle intervention among people with IGT reduced the development of visionthreatening retinopathy by $47 \%$ over a 20 year interval. This reduction occurred in conjunction with a $43 \%$ reduction in the incidence of diabetes [2] and represents the first evidence from a randomised controlled trial that lifestyle interventions which reduce the incidence of diabetes may reduce the incidence of diabetes-related complications. Using lifestyle interventions over a 6 year period, the number with IGT needed to treat to prevent one case of proliferative retinopathy or blindness was 14 persons $(95 \%$ CI 9-205).

Proliferative retinopathy and blindness occurred only among those who had already developed diabetes, predominantly among those who had had diabetes for 10 years or more, indicating the specificity of this microvascular complication. The 20 year cumulative incidence of severe retinopathy of $16.2 \%$ in the control group affirms the high risk of this complication among people with IGT who subsequently develop diabetes. In contrast, NPR assessed among the 20 year survivors was equally prevalent in the intervention and control groups. While significantly more frequent in those with diabetes $(41.6 \%)$, NPR was also seen in $14.3 \%$ of those who did not develop diabetes, confirming that it is a common but less specific microvascular complication than $\operatorname{PR}[22,23]$. None of the participants with IGT had proliferative retinopathy at the inception of the intervention trial. Although there are only limited data, the prevalence of severe retinopathy in Chinese patients with diabetes is reported to be higher than in other ethnic groups [24].

Several factors may explain why we observed effects on severe retinopathy but not on nephropathy and neuropathy. First, the low cumulative incidence of severe nephropathy and neuropathy limited the statistical power to detect differences and may limit conclusions about whether or not lifestyle intervention influences these complications. Severe nephropathy, resulting in the need for renal replacement treatment or death, was found in only nine $(1.7 \%)$ participants, and abnormal renal function, defined as ACR $\geq$ $300 \mathrm{mg} / \mathrm{g}$ or elevated serum creatinine, was present in only $4.2 \%$ of the surviving participants $(5.1 \%$ in those with and $0 \%$ in those without diabetes). Severe neuropathy was also rare, affecting $1.2 \%$ of intervention and $1.5 \%$ of control participants. The relatively low frequencies of severe nephropathy and neuropathy may be attributable to the relatively short duration of diabetes in many participants, as most longitudinal studies report a marked increase in the incidence of severe nephropathy only after a duration of diabetes of 15-20 years or more [25, 26]. Second, nephropathy based on albumin excretion and neuropathy based on the monofilament test were assessed only at follow-up in living participants; thus, we lacked information on the $25 \%$ who died during the study. This means that the findings could have been affected by a survival bias and competing mortality, whereby people who had these complications were less likely to be identified, reducing the incidence and the ability to detect an intervention effect. Given these limitations, much larger studies, such as the US Diabetes Prevention Program Outcome Study [27], perhaps with even longer follow-up, are needed to investigate whether or not lifestyle intervention influences the incidence of these complications.

Finally, retinopathy may simply be more directly influenced by the protective effects of lifestyle-based diabetes prevention 
because retinopathy is more specific to diabetes than either neuropathy or nephropathy.

The follow-up study was designed to determine the longterm effects of lifestyle intervention on the incidence of diabetes, but with the prespecified secondary objective of determining its effect on the incidence of the common microvascular complications of retinopathy, nephropathy and neuropathy. The original study was not designed to examine the effects of intervention on vascular complications and thus the statistical power of the follow-up study to detect differences in these complications was limited. As the three original active treatment groups showed similar reductions in diabetes incidence, they were combined in the follow-up study to attempt to overcome this limitation. The study used cluster randomisation of clinics, with all participants in each individual clinic assigned to receive the same intervention. This design greatly facilitated the delivery of group instruction as all participants and staff in the same clinic received and delivered the same message, thereby minimising the likelihood of contamination among the intervention groups. The statistical analyses were adjusted for cluster randomisation to account for this feature of the study design. Clinically overt microvascular events were ascertained from interviews using standardised questionnaires and physical examinations, and from medical chart reviews conducted by personnel who were not aware of the participant's treatment assignment, thus alleviating the likelihood of interviewer or observer biases. As participants were not systematically tested for clinically asymptomatic microvascular complications throughout the study, recognition of these complications depended on the 20 year followup examinations carried out among $365(91 \%)$ of the 400 survivors, who represented only $69 \%$ of the original participants. A possible source of differential bias is that as diabetes was more frequent among the control group, a greater proportion of participants in this group was likely to have had routine ophthalmoscopic examinations to detect retinopathy. However, as severe retinopathy was found exclusively among participants who had already developed diabetes, a greater proportion of ophthalmological examinations among the controls than among the intervention group seems an unlikely source of such bias. While the proportions of survivors examined in the intervention and control groups (91 and 93\%, respectively) were similar, results from these examinations require cautious interpretation as the participants were not necessarily representative of those originally assigned to these groups and were subject to survivor bias and the effects of treatment for comorbid conditions. The absence of information on potential risk factors (other than diabetes duration), except at baseline and at the end of the active intervention, prevents inferences about mechanisms whereby lifestyle intervention other than by preventing diabetes may have reduced the incidence of severe retinopathy.
While the lower incidence of severe retinopathy was associated with a reduced incidence of diabetes in the intervention group, a mechanism other than mitigation of glycaemia might be responsible. However, an equivalent reduction in the incidence of severe retinopathy was seen among participants assigned to each of the three original intervention strategies. This suggests that the effects on the incidence of proliferative retinopathy were not dependent on a particular component of the intervention, such as dietary change or exercise. Nevertheless, the concomitant reductions in the incidences of retinopathy and diabetes could be due to factors other than the reduction in glycaemia per se.

This study showed that group lifestyle intervention in people with IGT resulted in a significantly reduced incidence of one of the most serious complications of diabetes - severe retinopathy. To our knowledge, this is a first demonstration that such interventions can reduce at least one of the more common serious microvascular complications of diabetes that is a major cause of morbidity in those with type 2 diabetes. The main reason for the lower incidence of retinopathy was the lower incidence of diabetes and the associated mean delay of 3.6 years in the onset of diabetes among participants who were assigned to the group-based intervention strategies [2].

We conclude that the lifestyle intervention delayed or prevented the onset of type 2 diabetes, and also that its effects delayed or prevented at least one of the serious microvascular complications of the disease. The $47 \%$ reduction in severe retinopathy together with the $17 \%$ reduction in cardiovascular disease mortality reported previously [2] provide a strong additional rationale for pursuing lifestyle intervention in persons with IGT and additional evidence to justify public health efforts to reduce the enormous global burden of type 2 diabetes using lifestyle interventions.

Acknowledgements We thank the participants in the original Da Qing Diabetes Prevention Study and the Da Qing Diabetes Prevention Outcome Study. We thank L. Zhang and H. Zhang of the China-Japan Friendship Hospital for grading the fundus photographs. We thank M. M. Engelgau for assistance in acquiring funding and B.V. Howard for help in the design of the original study. We also acknowledge L. Kong, the China Ministry of Health, the leadership of the ChinaJapan Friendship Hospital, Da Qing First Hospital, the Da Qing City Health Bureau and the Beijing and West Pacific Regional Office of WHO for their general support. We thank W.C. Knowler, C.C. Mason, R.G. Nelson and S. Kobes of the Diabetes Epidemiology and Clinical Research Section, NIDDK, for statistical assistance. Special thanks are due to the late Professor X. Pan as this study would not have been possible without his leadership in the original Da Qing Diabetes Prevention Study. This study was supported by CDC/WHO Cooperative Agreement No. U58/CCU424123-01-02, China-Japan Friendship Hospital and Da Qing First Hospital. Q. Gong received a Mentorbased Postdoctoral Research Fellowship from the American Diabetes Association and partial support from Intramural Research Program of the National Institute of Diabetes and Digestive and Kidney Diseases. The sponsors had no role in any aspect of the study design, in the 
collection, analysis and interpretation of data, or in the development of the manuscript.

Duality of interest The authors declare that there is no duality of interest associated with this manuscript.

\section{References}

1. Pan XR, Li GW, Hu YH et al (1997) Effects of diet and exercise in preventing NIDDM in people with impaired glucose tolerance. The Da Qing IGT and Diabetes Study. Diabetes Care 20:537-544

2. Li G, Zhang P, Wang J et al (2008) The long-term effect of lifestyle interventions to prevent diabetes in the China Da Qing Diabetes Prevention Study: a 20-year follow-up study. Lancet 371:1783-1789

3. Tuomilehto J, Lindstrom J, Eriksson JG et al (2001) Prevention of type 2 diabetes mellitus by changes in lifestyle among subjects with impaired glucose tolerance. N Engl J Med 344:1343-1350

4. Knowler WC, Barrett-Connor E, Fowler SE et al (2002) Reduction in the incidence of type 2 diabetes with lifestyle intervention or metformin. N Engl J Med 346:393-403

5. Ramachandran A, Snehalatha C, Mary S, Mukesh B, Bhaskar AD, Vijay V (2006) The Indian Diabetes Prevention Programme shows that lifestyle modification and metformin prevent type 2 diabetes in Asian Indian subjects with impaired glucose tolerance (IDPP-1). Diabetologia 49:289-297

6. Kosaka K, Noda M, Kuzuya T (2005) Prevention of type 2 diabetes by lifestyle intervention: a Japanese trial in IGT males. Diabetes Res Clin Pract 67:152-162

7. Gerstein HC, Yusuf S, Bosch J et al (2006) Effect of rosiglitazone on the frequency of diabetes in patients with impaired glucose tolerance or impaired fasting glucose: a randomised controlled trial. Lancet 368:1096-1105

8. Preis SR, Hwang SJ, Coady S et al (2009) Trends in all-cause and cardiovascular disease mortality among women and men with and without diabetes mellitus in the Framingham Heart Study, 1950 to 2005. Circulation 119:1728-1735

9. Morrish NJ, Wang SL, Stevens LK, Fuller JH, Keen H (2001) Mortality and causes of death in the WHO Multinational Study of Vascular Disease in Diabetes. Diabetologia 44(Suppl 2): S14-S21

10. Gregg EW, Gu Q, Cheng YJ, Narayan KM, Cowie CC (2007) Mortality trends in men and women with diabetes, 1971 to 2000. Ann Intern Med 147:149-155

11. Klein R, Klein BE, Moss SE, Davis MD, DeMets DL (1984) The Wisconsin epidemiologic study of diabetic retinopathy. II. Prevalence and risk of diabetic retinopathy when age at diagnosis is less than 30 years. Arch Ophthalmol 102:520-526

12. Klein R, Klein BE, Moss SE, Davis MD, DeMets DL (1984) The Wisconsin epidemiologic study of diabetic retinopathy. III.
Prevalence and risk of diabetic retinopathy when age at diagnosis is 30 or more years. Arch Ophthalmol 102:527-532

13. Pavkov ME, Bennett PH, Sievers ML et al (2005) Predominant effect of kidney disease on mortality in Pima Indians with or without type 2 diabetes. Kidney Int 68:1267-1274

14. Nelson RG, Pavkov ME, Hanson RL, Knowler WC (2008) Changing course of diabetic nephropathy in the Pima Indians. Diabetes Res Clin Pract 82(Suppl 1):S10-S14

15. Gregg EW, Sorlie P, Paulose-Ram R et al (2004) Prevalence of lower-extremity disease in the US adult population $\geq 40$ years of age with and without diabetes: 1999-2000 national health and nutrition examination survey. Diabetes Care 27:1591-1597

16. Hu YH, Pan XR, Liu PA, Li GW, Howard BV, Bennett PH (1991) Coronary heart disease and diabetic retinopathy in newly diagnosed diabetes in Da Qing, China: the Da Qing IGT and Diabetes Study. Acta Diabetol 28:169-173

17. Anonymous (1991) Grading diabetic retinopathy from stereoscopic colour fundus photographs - an extension of the modified Airlie House classification. ETDRS report number 10. Early Treatment Diabetic Retinopathy Study Research Group. Ophthalmology 98:786-806

18. Boulton AJ, Malik RA, Arezzo JC, Sosenko JM (2004) Diabetic somatic neuropathies. Diabetes Care 27:1458-1486

19. Anonymous (1985) Diabetes mellitus Report of a WHO Study Group. World Health Organ Tech Rep Ser 727:1-113

20. Pearson ES, Hartley HO (1966) Biometrika tables for statisticians, 3rd edn. Cambridge University Press, Cambridge

21. Altman DG, Andersen PK (1999) Calculating the number needed to treat for trials where the outcome is time to an event. BMJ 319:1492-1495

22. Kempen JH, O'Colmain BJ, Leske MC et al (2004) The prevalence of diabetic retinopathy among adults in the United States. Arch Ophthalmol 122:552-563

23. Diabetes Prevention Program Research Group (2007) The prevalence of retinopathy in impaired glucose tolerance and recent-onset diabetes in the Diabetes Prevention Program. Diabetes Prevention Program Research Group. Diabet Med 24:137-144

24. Chi ZS, Lee ET, Lu M, Keen H, Bennett PH, WHO Multinational Study Group (2001) Vascular disease prevalence in diabetic patients in China: standardised comparison with the 14 centres in the WHO Multinational Study of Vascular Disease in Diabetes. Diabetologia 44(Suppl 2):S82-S86

25. Nelson RG, Newman JM, Knowler WC et al (1988) Incidence of end-stage renal disease in type 2 (non-insulin-dependent) diabetes mellitus in Pima Indians. Diabetologia 31:730-736

26. Kunzelman CI, Knowler WC, Pettitt DJ, Bennett PH (1989) Incidence of proteinuria in type 2 diabetes mellitus in the Pima Indians. Kidney Int 35:681-687

27. Diabetes Prevention Program Research Group, Knowler WC, Fowler SE et al (2009) 10-Year follow-up of diabetes incidence and weight loss in the Diabetes Prevention Program Outcomes Study. Diabetes Prevention Program Research Group. Lancet 374:1677-1686 